\title{
The multiplier role of psychiatrists in low income settings
}

\author{
F. Kigozi* and J. Ssebunnya \\ Butabika National Referral and Teaching Mental Hospital, Kampala, Uganda
}

\begin{abstract}
Mental health care is receiving increased attention in low-income countries with the availability of a wide range of effective evidence-based treatments for acute and chronic mental disorders amidst scarce resources. Availability of these treatments and competent human resources enables the use of a variety of interventions at several levels of care for persons with mental illness and makes it feasible to ensure observance of quality in the treatment approaches that go beyond institutionalisation. However, unlike developed countries which are endowed with many and relatively well-paid mental health specialists, low-income countries face a dire shortage of highly trained mental health professionals in addition to several other challenges. In light of this, there is need to re-assess the role of the few available psychiatrists, with a shift to new core tasks such as designing mental health care programmes that can be delivered by non-specialists, building their health system's capacity for delivering care, including supporting front-line health workers through support supervision, raising awareness on mental health and patients' rights in addition to promoting essential research. This requires a fundamental paradigm shift from the current training for mental health specialists to a public health oriented approach and providing incentives for community engagement.
\end{abstract}

First published online 27 February 2014

Key words: Low-income countries, mental health care, primary health care, psychiatrist.

\section{Introduction}

Neuropsychiatric conditions comprise $14 \%$ of the global burden of disease and $30 \%$ of all noncommunicable diseases (Prince et al. 2007). About one in four people will have a mental health problem at some time in their lives. For some people, this will be a short period of mild depression, anxiety or stress. For others, it will be a serious, long-lasting condition such as schizophrenia, bipolar disorder or major depression (McBain et al. 2012). These disorders are associated with premature mortality and profound socio-economic impacts on individuals, with substantial costs to families and the country (Patel, 2009).

An estimated $80 \%$ of persons with mental illnesses live in low-and middle-income countries (LAMICs) (Prince et al. 2007; McBain et al. 2012) and yet because of high rates of infectious diseases, those countries that are the poorest typically have the lowest expenditures on mental health care, with the majority spending less than $1 \%$ of their total health budgets on mental health. The majority of these countries also greatly rely on a framework of institutionalised care (World Health Organization, 2005; Saxena et al. 2006a). For example,

\footnotetext{
* Address for correspondence: Dr F. Kigozi, Butabika National Referral and Teaching Mental Hospital, Kampala-Uganda, P.O Box 7017, Kampala, Uganda.

(Emails: fredkigozi@yahoo.com; joy95h@yahoo.co.uk)
}

only $52 \%$ of low-income countries, as compared to $97 \%$ of high-income countries, provide communitybased care for patients (Saxena et al. 2007). This dearth of financial resources directed towards mental health is also associated with a shortage of mental health human resources in both primary and tertiary care. (Patel, 2009; Bhana et al. 2010; Patel et al. 2011).

\section{Mental health care and the treatment gap in low-income countries}

Despite the existence of cost-effective interventions, the number of persons who remain untreated is estimated at $85 \%$ in LAMICs, leading to chronicity, suffering and increased costs of care (McBain et al. 2012). Access to psychotropic medicines and psychosocial interventions varies greatly across these countries, with marked inequity even within countries. Sadly, the evidence concerning the substantial burden of mental disorders has not been translated into adequate investments in mental health care thus making the treatment gap more pronounced in LAMICs (Babor et al. 1994; World Health Organization, 2005).

Progress in mental health service development has been slow in most low-income countries due to a number of barriers and challenges as documented in several studies. These among others include: a high burden of mental disorders, absence of political will resulting in low prioritisation for mental health, absence of (or outdated) 
policies, plans and laws; shortage and skewed distribution of the few available specialists, general health workers who are often inadequately trained to handle mental health problems, brain drain, limited public awareness about mental health and poor service utilisation (Saraceno et al. 2007; Patel, 2009; Thornicroft et al. 2010; Lund et al. 2011; Doku et al. 2011; Mwanza et al. 2011; Ssebunnya et al. 2011).

The impact of these barriers is indeed greatest in low- and middle-income countries, where the same challenges and barriers are greatest, with many competing claims on the available limited resources.

\section{Current mental health care and resources in LAMICs}

Modern psychiatric services do not exist for the largest proportion of the population in developing countries, which is predominantly rural. The few psychiatrists in these countries are usually based in urban settings and are primarily concerned with hospital-based care for those with severe mental illness, leaving the majority of the rural population with little or no care at all. (Patel \& Winston, 1994).

The WHO Mental Health Atlas has reviewed global mental health resources indicating that despite the majority of global burden of mental disorders being located in LAMICs, these countries have less than $10 \%$ of global mental health resources, with the median number of psychiatrists in high-income countries found to be 200 times greater than that in low-income countries (Bruckner et al. 2011). Despite this disparity, some high-income countries continue to rely on the already meagre mental health resources migrating from LAMICs to sustain their own mental health systems resulting in marked brain drain (Patel, 2003).

Integrating mental health care into primary care through 'task-sharing' has been proposed as part of the mental health strategy to address the gap created by the acute shortage of specialists. The core objective of the strategy is a rational redistribution of tasks among health workforce teams that involves the delegation of mental health care to non-specialist health workers who are trained to deliver interventions for specific mental disorders, thereby improving the human resources workforce skills and distribution (Lancet Global Mental Health Group, 2007; Petersen et al. $2011 a$, b; Patel et al. 2013). The strategy has been advocated and indeed is being applied in some countries to provide access to mental health care on a wide scale.

\section{The role of psychiatrists}

Several developing countries have undertaken various innovations in mental health service delivery to ensure that the enormous gap between the need and availability of services is narrowed (Srinivasa, 1998). Since the Alma Ata declaration and the WHO (2001) report, several countries embraced the Primary Health Care approach and have been implementing integration of mental health into primary health care, with varying degree of success in improving access and equity in mental health service delivery (World Health Organization, 2001; Kigozi, 2007; Kigozi \& Ssebunnya, 2009; Ssebunnya et al. 2010).

To achieve the intended objectives of this approach, deliberate programme design is essential, including regular human resource training, clear referral pathways and support network; targeting those in the primary care setting.

The psychiatrist will for the foreseeable future remain an expert clinician for service delivery both at primary and tertiary levels, with key roles of clinical assessment, diagnosing and treating persons with mental illness; training fellow mental health professionals as well as developing and disseminating guidelines for fellow professionals (Farooq \& Minhas, 2001; Patel, 2009). This should be in addition to other key roles with multiplier effects such as influencing resource mobilisation, generating demand for service utilisation and contributing to the provision of an enabling professional environment for scaling up mental health services. Thus, it is increasingly becoming clear that for mental health services to develop, there is need to transform the role of the few existing mental health specialists in low income settings from a purely service-delivery approach to public mental health leadership (Lancet Global Mental Health Group, 2007). The psychiatrist's new roles as a leader of a multidisciplinary team should include designing and managing such programmes for enhancing capacity for mental health service delivery in consultation with other professionals and lay workers in primary care, supervision of junior staff and trainees, ensuring quality care and providing referral pathways (Patel, 2009).

Psychiatrists are seen to possess a unique expertise, and occupy leading positions in most countries, functioning as advisers to governments and chairing key committees that are responsible for the formulation of mental health policies, action plans and legislations to improve the mental health systems (Thornicroft et al. 2010). They have therefore a unique opportunity and role to shape the process of reform in the best interest of patients, families and carers, the public and fellow health workers (Muijen, 2006).

$\mathrm{He} / \mathrm{she}$ must develop skills that may not be included in the traditional teaching, especially for service development. They therefore need to play a public mental health leadership role in increasing the coverage of mental health care through task shifting of effective 
interventions to non-specialist health workers. In view of the limited mental health awareness and poor utilisation of the existing services, such programmes should also focus on increasing population awareness on mental health and mental illness, the rights of people with mental illness and available treatments so as to increase levels of help-seeking and fighting stigma. Furthermore, there is a need to focus on improving awareness of benefits of accessing facilities and services (Thornicroft et al. 2010).

Human resources are the most critical asset in mental health service provision. In many developing countries, trained psychiatrists work under conditions of heavy and relentless clinical activities, and may not have dedicated time during the week for any service development duties.

One of the important recommendations for human resource development is the increasing emphasis on integration of mental health into primary care setting, thereby increasing access to the vast majority of the underserved population. In view of the shortage of mental health professionals in developing countries, there is a need for well-rounded generalists who are capable of coping with most psychiatric problems, with little or no access to any mental health practitioner. This mandates the few available specialists to train general health workers in basic skills in mental health care, such as detection of mental disorders, provision of basic care and referral of complex problems for specialist care; which skills are best given by psychiatrists (Thornicroft et al. 2010). Appropriate training also requires being combined with continued supervision and support to achieve effective mental health care in PHC settings (World Health Organization, 2003). As a teacher and clinical consultant in mental health, the psychiatrist therefore has to play a training and supervisory role for existing general health workers as well as other junior mental health professionals.

The need to foster a public health approach to mental health service delivery is becoming more pronounced, which necessitates involving psychiatrists as experts in planning, education and overcoming inertia and resistance in the current environment. This may also involve influencing key leaders into re-designing and monitoring the organisation of the mental health system (Lancet Global Mental Health Group, 2007; Patel, 2009). As a highly respectable professional, particularly in LAMICs, the psychiatrist can play a big role in conducting mental health advocacy and engaging policy makers for prioritising mental health and increasing provision of resources. The advocacy could be done through avenues such as designing media programmes, with a net effect of creating demand for utilisation of services.

The needs of patients and families in modern mental health practice are beyond the capacity of a single sector. Strong links between psychiatrists, community leaders, patients and families, and other stakeholders are vital for progress. When strong partnerships exist, they can contribute to the community understanding and advancement of mental health issues, as the first step towards scaling up good quality care for those living with mental illnesses, prevention of illnesses, and promotion of mental health through collaboration with other sectors. Psychiatrists should therefore build linkages with NGOs and other partners involved in mental health service delivery (Muijen, 2006).

Further, studies have highlighted the need for mental health promotion and disease prevention through broader influences in both health and non-heath sectors such as education, employment and other community sectors. Improved mental health can in turn assist these sectors with their own outcomes (Beddington et al. 2008; Herrman et al. 2008). Psychiatrists should be at the forefront in recommending strategies for promoting mental health in these sectors as well as supporting the development of partnerships (Saxena et al. 2006b).

Creating databases to organise information to aid and/or inform policy development is very crucial for service development. This necessitates conceptualising, directing and conducting mental health research as well as dissemination of research output, in which psychiatrists are expected to play a crucial role as well (Farooq \& Minhas, 2001). Further, as part of capacity building and mentoring, psychiatrists must inculcate a research oriented approach/culture at all levels of service delivery; sharing out challenges, solutions and new discoveries. Psychiatrists are well trained and have the abilities to synthesise materials from various sources, which should be shared with the lower level frontline health workers, thereby increasing knowledge and application of evidencebased approach to mental health care. This improves aspects of quality assurance practice in LAMICs; which has for a long time been a preserve of higher institutions of care in big cities and universities and yet equally important for the huge number of lower level health workers in the system.

However, it should be noted that the new additional roles being advocated for the psychiatrists may be complex, and to be effective requires a good grasp of the needs of patients in multiple settings and the ability to work intensively with staff from a variety of backgrounds. Although the essential role of most psychiatrists could remain therapeutic interventions with patients, the type of mental health problems presenting in the community setting and the expectations of psychiatrists' role will be radically different, rendering a detached medical role insufficient (Muijen, 2006). 


\section{Conclusion}

Developing countries face a dire shortage of mental health resources especially at the specialist psychiatrist level. The situation is worsened by the current unplanned population explosion, with sudden changes in the lifestyle and social support network, uneven growth, inequity in resource distribution and high burden of disease. Although the psychiatrist's primary role has been mainly that of offering clinical management and consultation, it is increasingly becoming clear that this has to fundamentally change so as to give the much needed key leadership role in the LAMIC's quest to narrow the treatment gap by generating a critical mass of relatively well-skilled mental health resources. The psychiatrist should thus provide a multiplier role at several levels of health service delivery with generation of adequately equipped mental health resources, stimulate demand for service utilisation and improve human rights observance within a conducive environment of appropriate legal context, policies and programme designs so as to meet the mental health needs of the population.

\section{Financial Support}

This research received no specific grant from any funding agency, commercial or not-for-profit sectors.

\section{Conflict of Interest}

The authors declare that there are no conflicting interests with their scientific work.

\section{References}

Babor TF, Grant M, Acuda W, Burns FH, Campillo C, Del Boca FK, Hodgson R, Ivanets NN, Lukomskya M, Machona M, Rollnick S, Resnick R, Saunders JB, Skutle A, Connor K, Ernberg G, Kranzler H, Lauerman R, McRee B (1994). A randomized clinical trial of brief interventions in primary care: summary of a WHO project. Addiction 89, 657-660; discussion 660-678.

Beddington J, Cooper CL, Field J, Goswami U, Huppert FA, Jenkins R, Jones HS, Kirkwood TB, Sahakian BJ, Thomas SM (2008). The mental wealth of nations. Nature 455, 1057-1060.

Bhana A, Petersen I, Baillie KL, Flisher AJ, The MHaPP Research Programme Consortium (2010). Implementing the World Health Report 2001 recommendations for integrating mental health into primary health care: a situation analysis of three African countries: Ghana, South Africa and Uganda. International Review of Psychiatry 22, 599-610.

Bruckner TA, Scheffler RM, Shen G, Yoon J, Chisholm D, Morris J, Fulton BD, Dal Poz MR, Saxena S (2011). The mental health workforce gap in low- and middle-income countries: a needs-based approach. Bulletin of the World Health Organization 89, 184-194.

Doku V, Ofori-Atta A, Akpalu B, Osei A, Read U, Cooper S, the MHaPP Research Programme Consortium (2011). Stakeholders' perceptions of the main challenges facing Ghana's mental health care system: a qualitative analysis. International Journal of Culture and Mental Health 4, 8-22.

Farooq S, Minhas FA (2001). Community psychiatry in developing countries - a misnomer? Psychiatric Bulletin 25, 226-227.

Herrman H, Moodie R, Saxena S (2008). Mental health promotion. In International Encyclopedia of Public Health (ed. K Heggenhougen and Q Stella), pp. 406-418. Academic Press: San Diego.

Kigozi F (2007). Integrating mental health into primary health care. Uganda's experience. South African Psychiatry Review 10, 17-19.

Kigozi FN, Ssebunnya J (2009). Integration of mental health into primary health care in Uganda: opportunities and challenges. Mental Health in Family Medicine 6, 37-42.

Lancet Global Mental Health Group (2007). Scaling up services for mental disorders: a call for action. Lancet 370, 1241-1252.

Lund C, Kleintjes S, Cooper S, Petersen I, Bhana A, Flisher AJ, the MHaPP Research Programme Consortium (2011). Challenges facing South Africa's mental health care system: stakeholders' perceptions of causes and potential solutions. International Journal of Culture and Mental Health 4, 23-38.

McBain R, Norton DJ, Morris J, Yasamy MT, Betancourt TS (2012). The role of health systems factors in facilitating access to psychotropic medicines: a cross-sectional analysis of the WHO-AIMS in 63 Low- and Middle-Income Countries. PLoS Medicine 9, e1001166.

Muijen M (2006). Challenges for Psychiatry: delivering the mental health declaration for Europe. World Psychiatry 5, 113-117.

Mwanza J, Cooper S, Kapungwe A, Sikwese A, Mwape L, the MHaPP Research Programme Consortium (2011). Stakeholders' perceptions of the main challenges facing Zambia's mental health care system: a qualitative analysis. International Journal of Culture and Mental Health 4, 39-53.

Patel V (2003). Recruiting doctors from developing countries: the great brain robbery? British Medical Journal 327, 926-928.

Patel V (2009). The future of psychiatry in low- and middle-income countries. Psychological Medicine 39, 1759-1762.

Patel V, Winston M (1994). The 'universality' of mental disorder revisited: assumptions, artifacts and new directions. British Journal of Psychiatry 165, 437-440.

Patel V, Maj M, Flisher AJ, De Silva MJ, Koschorke M, Prince M, WPA zonal and member society representatives (2011). Reducing the treatment gap for mental disorders: a WPA survey. World Psychiatry 9, 169-176.

Patel V, Belkin GS, Chockalingam A, Cooper J, Saxena S, Unützer J (2013). Grand Challenges: Integrating Mental Health Services into Priority Health Care Platforms. PLoS Medicine 10, e1001448.

Petersen I, Lund C, Bhana A, Flisher AJ (2011a). A task-shifting approach to primary mental health care for 
adults in South Africa: human resource requirements and costs for rural settings. Health Policy and Planning 10, 1093.

Petersen I, Ssebunnya J, Bhana A, Baillie K, MhaPP Research Programme Consortium (2011b). Lessons from case studies of integrating mental health into primary health care in South Africa and Uganda. International Journal of Mental Health Systems 15, 5-8.

Prince M, Patel V, Saxena S, Maj M, Maselko J, Phillips MR, Rahman A (2007). No health without mental health. Lancet 370, 859-877.

Saraceno B, van Ommeren M, Batniji R, Cohen A, Gureje O, Mahoney J, Sridhar D, Underhill C (2007). Barriers to improvement of mental health services in low-income and middle-income countries. Lancet 370, 1164-1174.

Saxena S, Sharan P, Garrido M, Saraceno B (2006a). World Health Organization's Mental Health Atlas 2005: implications for policy development. World Psychiatry 5, 179-184.

Saxena S, Paraje G, Sharan P, Karan G, Sadana R (2006b). The 10/90 divide in mental health research: trends over a 10-year period. British Journal Psychiatry 188, 81-82.

Saxena S, Thornicroft G, Knapp M, Whiteford H (2007). Resources for mental health: scarcity, inequity, and inefficiency. Lancet 370, 878-889.

Srinivasa MR (1998). Rural psychiatry in developing countries. Psychiatric Services 49, 967-969.
Ssebunnya J, Kigozi F, Kizza D, Ndyanabangi S, MHAPP Research Programme Consortium (2010). Integration of mental health into primary health care in a rural district in Uganda. African Journal of Psychiatry 13, 128-131.

Ssebunnya J, Kigozi F, Ndyanabangi S, Cooper S (2011). Stakeholders' perceptions of the main challenges facing Uganda's mental health care system: a qualitative analysis. International Journal of Culture and Mental Health $\mathbf{4}$, 54-67.

Thornicroft G, Alem A, Antunes Dos Santos R, Barley E, Drake RE, Gregorio G, Hanlon C, Ito $\mathrm{H}$, Latimer E, Law A, Mari J, McGeorge P, Padmavati R, Razzouk D, Semrau M, Setoya Y, Thara R, Wondimagegn D (2010). WPA guidance on steps, obstacles and mistakes to avoid in the implementation of community mental health care. World Psychiatry 9, 67-77.

World Health Organization (2001). The World Health Report 2001. Mental Health: New Understanding, New Hope. WHO: Geneva.

World Health Organization (2003). WHO Module on Human Resources and Training in Mental Health 2003 WHO: Geneva.

World Health Organization (2005). Mental Health Atlas 2005. WHO: Geneva. 